\title{
Genomic re-assessment of the transposable element landscape of the potato
} genome

\author{
Diego Zavallo*1, Juan Manuel Crescente ${ }^{\star 2,4}$, Magdalena Gantuz ${ }^{3,4}$, Melisa Leone ${ }^{1,5}$, \\ Leonardo Sebastian Vanzetti ${ }^{2,4}$, Ricardo Williams Masuelli ${ }^{3,4}$, and Sebastian Asurmendi ${ }^{1}$ \\ ${ }^{1}$ Instituto de Agrobiotecnología y Biología Molecular (IABIMO), Instituto Nacional de \\ Tecnología Agropecuaria (INTA), Consejo Nacional de investigaciones Científicas y \\ Tecnológicas (CONICET), Los Reseros y Nicolas Repeto, Hurlingham, Argentina \\ ${ }^{2}$ Grupo Biotecnologia y Recursos Genéticos, EEA INTA Marcos Juárez, Ruta 12 km 3, \\ 2580 Marcos Juárez, Argentina \\ ${ }^{3}$ Instituto de Biología Agrícola de Mendoza (IBAM), Facultad de Ciencias Agrarias \\ (FCA), CONICET-UNCuyo, Almirante Brown 500, M5528AHB, Chacras de Coria, \\ Mendoza, Argentina \\ ${ }^{4}$ Consejo Nacional de Investigaciones Científicas y Técnicas (CONICET), Argentina \\ ${ }^{5}$ Agencia Nacional de Promocion Científica y Tecnológica (ANPCyT), Argentina. \\ * Both authors contributed equally to this work \\ Corresponding author: \\ Diego Zavallo: E-mail: zavallo.diego@inta.gob.ar; Phone: (5411) 4621 447/1676, int. \\ 3526. Orcid No: 0000-0002-9021-2175 \\ Sebastian Asurmendi: E-mail: asurmendi.sebastian@inta.gob.ar; Phone: (5411) 4621 \\ 447/1676, int. 3639. Orcid No: 0000-0001-9516-5948
}

\section{Abstract}

Transposable elements (TEs) are DNA sequences with the ability to auto-replicate and move throughout the host genome. TEs are major drivers in stress response and genome evolution. Given their significance, the development of clear and efficient TE annotation pipelines has become essential for many species. The latest de novo TE discovery tools, along with available TEs from Repbase and sRNA-seq data, allowed us to perform a reliable potato TEs detection, classification and annotation through an open -source and freely available pipeline (https://github.com/DiegoZavallo/TE_Discovery). Using a variety of tools, approaches and rules, our pipeline revealed that ca. $16 \%$ of the potato genome can be clearly annotated as TEs. Additionally, we described the distribution of the different types of TEs across the genome, where LTRs and MITEs present a clear clustering pattern in pericentromeric and subtelomeric/telomeric regions respectively. Finally, we analyzed the insertion age and distribution of LTR retrotransposon families which display a distinct pattern between the two major 
superfamilies. While older Gypsy elements concentrated around heterochromatic regions, younger Copia elements located predominantly on euchromatic regions. Overall, we delivered not only a reliable, ready-to-use potato TE annotation files, but also all the necessary steps to perform de novo detection for other species.

Keywords: Transposable elements; Solanum tuberosum; potato; TEs annotation; Retrotransposons; DNA transposons

\section{Key Message}

We provide a comprehensive and reliable potato TE landscape, based on a wide variety of identification tools and integrative approaches, producing clear and ready-touse outputs for the scientific community.

\section{Introduction}

Transposable elements (TEs) are DNA sequences with the ability to auto-replicate and move throughout the genome. In plants, TEs can occupy a large proportion of the genome, representing more than half of the total genomic DNA in some cases. For example, they comprise about $85 \%$ and $88 \%$ of wheat and maize genomes, respectively (Schnable et al., 2009; Appels et al., 2018) which may indicates the relevance of these elements to genome architecture and size (Roessler et al., 2018).

Furthermore, over the past few years an increasing number of studies have shed light on their importance in gene regulation (Hirsch and Springer, 2017; Judd and Feschotte, 2018) stress response and genome evolution (Hosaka and Kakutani, 2018).

TEs are usually divided into two major classes based on their mechanism of transposition. Class I elements (or retrotransposons) propagate via a reversetranscribed RNA intermediate, whereas class II elements (or DNA transposons) move through a "cut and paste" mechanism. Another type of classification is by their ability to transpose on their own (i.e. autonomous), characteristic shared by some TEs from both classes. The non-autonomous elements can move but rely on autonomous TEs for their mobility (Wicker et al., 2007).

Given their significance, the identification, classification and annotation of TEs has emerged as a new field of great interest in science, which involves both wet-lab biology and bioinformatics. As Hoen et al. (2015) describe in their review on TE annotation benchmarking, precise detection and annotation of TEs is a difficult task due to their great diversity, both within and among genomes. TEs differ across multiple attributes, including transposition mechanism, sequence, length, repetitiveness, and chromosomal 
82 distribution. In addition, whereas recently inserted TEs have a relatively low variability

83 within the family, over time they accumulate mutations and diverge, making them harder

84 to detect (Hoen et al., 2015).

There are two main strategies for TE annotation: homology-based and de novo identification, which can also be referred to as library-based and signature-based, respectively. The homology-based strategy uses libraries of known TEs such as the Repbase repository (Jurka et al., 2005) to screen genomes in order to identify similar sequences, most commonly by using RepeatMasker (Smit et al., 1996). On the other hand, de novo approaches use characteristic structural features, such as LTRs (Long Terminal Repeats) for retrotransposons and TIRs (Terminal Inverted Repeats) for DNA transposons, to identify new elements. Moreover, autonomous TEs have conserved structures like RT (reverse transcriptase) or TR (transposase) that can also be used for accurate TE identification (Wicker et al., 2007). Several tools based on structural features, such as LTRharvest (Ellinghaus et al., 2008) and TIRvish command, which are part of the GenomeTools suite (Gremme et al., 2013), are available. Other tools based on this criteria are specifically designed to discover different types of TE families (e.g. SINE Scan (Mao and Wang, 2016), HelitronScanner (Xiong et al., 2014) and MITE Tracker (Crescente et al., 2018)). Another de novo based strategy relies on the most important biological mechanism that silences TEs - RNA directed DNA Methylation (RdDM) - in which double-stranded RNAs (dsRNAs) are processed into 21-24 nt small interfering RNAs (siRNAs) and guide methylation on homologous DNA loci. For instance, TASR (for Transposon Annotation using Small RNAs) tool uses sRNAs Illumina data as guide for TE annotation/identification (El Baidouri et al., 2015). However, complete and accurate TEs annotation will likely require a combination of both homology-based and de novo methods together with an additional manual curation step (Platt et al., 2016).

Nevertheless, all of these tools often give a representative sequence for each family (usually a full-length TE sequence), but fail presenting their copies across the genome, not only for other potentially autonomous copies, but also for members that are partially or entirely deficient in one or more domains. Furthermore, in the case of nonautonomous TEs (e.g. SINEs, TRIMs and MITEs), the amount of copies across the genome is high due to their repetitive content and their short length; however this information is usually not presented.

115 For the scientific community, especially in genomics, the need for reliable annotation 116 is becoming fundamental. Generally, for each genome sequencing project, annotations 117 consisting mainly of protein-coding genes (structural and functional) and miRNAs genes 118 are made, whereas TEs remain poorly annotated As an example, the Gramene 
119 Database (http://www.gramene.org) is a curated, open-source database for comparative

120 functional genomics in crops and model plant species with information on more than two

121 million genes from 67 plant genomes. By contrast, the PGSB Repeat Element Database

122 (Nussbaumer et al., 2012) which compiles publicly available repeat sequences from

123 TREP (Wicker et al., 2002), TIGR repeats (Ouyang and Buell, 2004), PlantSat (Macas

124 et al., 2002), Genbank and de novo detected LTR retrotransposon sequences from

125 LTR_STRUC (McCarthy and McDonald, 2003) only comprises 62.000 sequences.

126 Nonetheless, in the latest version of sunflower genome, Badouin et al. (2017) performed

127 a comprehensive search for repeat elements by developing a new tool called Tephra

128 (https://github.com/sestaton/tephra) (Staton, 2018), which discovers and annotates all

129 types of transposons. Tephra combines existing specific transposon discovery tools for

130 all types of TEs, classifies and annotates them, but still lacks information on copy

131 numbers across the genome. A recent study addressed this issue by applying a method

132 called "Russian doll" due to its nesting strategy. This method builds nested libraries

133 establishing different search rules for each one of them. The first one includes only

134 "potentially autonomous TEs", the second one contains "total TEs", including non-

135 autonomous and a third one that also includes uncategorized "repeated elements"

136 (Berthelier et al., 2018).

137 Solanum tuberosum, the cultivated potato, is the third most important food crop after

138 rice and wheat, and the main horticultural crop (Devaux et al., 2014). The sequencing of

139 the $S$. tuberosum genome resulted in an assembly of $727 \mathrm{Mb}$ of $810.6 \mathrm{Mb}$ sequenced.

140 Because most potato cultivars are autotetraploid $(2 n=4 x=48)$ and highly heterozygous,

141 sequencing was performed on a homozygous doubled-monoploid potato clone. The

142 latest potato genome assembly (4.03) contains 39.031 annotated genes and $62.2 \%$ of

143 the genome corresponds to repetitive elements at scaffold level (Consortium et al., 144 2011).

145 Many attempts have been made to discover repetitive elements in Solanaceae 146 families. Most of these studies were mainly focused on tandem repetitive elements, 147 whereas studies of complex repetitive elements were mostly performed on limited 148 groups of TEs (Mehra et al., 2015). The researchers assessed the complex repetitive 149 elements in potato (S. tuberosum) and tomato (S. lycopersicum) genomes, identifying 150629,713 and 589,561 repetitive elements, respectively. Mehra et al. used 151 RepeatModeler (http://repeatmasker.org/RepeatModeler.html), which employs a 152 repetitiveness-based strategy, and enriched the amount of repeat families previously 153 identified in the 4.03 version of the potato genome with RepeatMasker (Smit et al., 154 1996). 
In this study we present an optimized pipeline of transposable elements detection and annotation from $S$. tuberosum. Our strategy relies on the combination of the latest de novo TE identification tools, available TEs from Repbase and Illumina sRNA-seq data to obtain TEs. We then find copies and applied a series of filters depending on the TE family to obtain a comprehensive and curated whole-genome atlas of potato transposable elements. Furthermore, we provide to the research community our pipeline, annotation results and files, ${ }^{-}$which are publicly available at https://github.com/DiegoZavallo/TE_Discovery to encourage reproducibility and the eventual implementation of our framework in diverse organisms.

\section{Materials and Methods}

\section{Input data}

The latest assembly version of Solanum tuberosum genome sequence was downloaded from the Potato Genome Sequencing Consortium (PGSC v4.03 of the doubled monoploid S. tuberosum Group Phureja DM1-3). Potato TEs sequences from Repbase Giri (Jurka et al., 2005) were downloaded prior registration. Note that LTRs transposons are divided in LTR (Long Terminal Repeat) and I (Internal) sequences, hence must be concatenated. We gathered 126 LTRs, 18 LINEs, 2 SINEs and 42 TIRs family sequences (https://www.girinst.org/repbase/update/search.php?query=tuberosum $\backslash \&$ querytype=Taxo noy).

Illumina sRNA-seq data for TASR run was generated by our lab (data unpublished); however, any available data from public repositories such as SRA (https://www.ncbi.nlm.nih.gov/sra) could be used as input.

\section{Transposable elements identification}

TEs obtained from different sources were merged together according to each element classification. Tephra, which uses several structure-based tools, was applied to harvest different kind of TEs in the potato genome. Tephra all command (which runs all subcommands) was executed using tephra config.yml file with default configuration parameters with the exception of $S$. tuberosum TEs from Repbase for the repeatdb parameter. A total of 1,325 Helitrons, 7,694 LTRs, 2,994 MITEs, 2,414 TIRs and 7,011 TRIMs families were found with this program. No non-LTR TEs (LINEs and SINEs) were found by Tephra. TASR (El Baidouri et al., 2015), a tool for de novo discovery of TEs using small RNA data, was also used in this work. 21, 22 and 24 nt sRNAs were parsed 
193 from files belonging to all treatments and replicates from our sRNA-Seq data and

194 subsequently concatenated to be used as input. TASR.v.1.1.pl perl script was run with

195 default parameters except for: -cpu 14, -nsirna 10, and-cnumber 5. A total of 1,916

196 families were found and presented as multifasta files comprising all the elements for

197 each family. A perl script provided by TASR developer was run in order to generate a

198 consensus sequence for each family. Then we created a single multifasta file with the

199 entire consensus. The PASTEC tool (Hoede et al., 2014) was used for this purpose,

200 since TASR does not classify TEs into categories. From the 1,916 families discovered, a

201 total of 891 LTRs, 49 LINEs, 15 SINEs, 9 TRIMs, 2 LARDs, 84 TIRs, 35 MITEs and 5

202 Helitrons were classified. For MITEs discovery, MITE Tracker (Crescente et al., 2018)

203 was employed using default parameters. A total of 1,045 MITEs elements were

204 detected. SINE_Scan (Mao and Wang, 2016), an efficient de novo tool to discover

205 SINEs was also used in this work with default parameters. A total of 13 SINEs families

206 were detected. As a result, we obtained 8,711 LTRs elements from Tephra, TASR and

207 Repbase, 67 LINEs elements from TASR and Repbase, 30 SINEs elements from TASR,

208 Repbase and SINE_Scan, 540 TIRs elements from Tephra, TASR and Repbase, 4,074

209 MITEs elements from Tephra, TASR and MITE Tracker and 1,330 Helitrons elements

210 from Tephra and TASR.

211

212

213

214

215

\section{Pipeline description}

To detect, filter and annotate TEs copies across the potato genome the obtained TEs list was subjected to an in house pipeline. The pipeline was developed using bash scripts and Jupyter notebooks.

1.1 add annotation.ipynb uses TEs sequences retrieved from each program and merge them together into one multi-fasta file per studied TE type (LTRs, LINEs, SINEs, TRIMs, LARDs, TIRs, MITEs and Helitrons). This script adds to each sequence a unique identifier containing an auto-incremented number, TE classification and the program source from which it was obtained.

2.1 vsearch.sh uses VSearch program to cluster similar sequences that share $80 \%$ identity, according to the $80 / 80 / 80$ rule in the study of Wicker et al. (2007). It is executed once per TE type, thus obtaining as a result TEs clustered by type.

2.2 vsearch merge.ipynb script uses vsearch outputs to create fasta files containing one TE per family. It also adds a family description indicating which program the members came from.

3.1 blast.sh performs a genome-wide BLASTn search using the files from the previous step and searches for TEs in the potato genome. For this task, the script uses 
230 the following parameters: -perc identity 80, -evalue 10e-3 and -task blasth (except for

231 LTRs). - qcov hsp perc 80 was used for SINEs, TRIMs and MITEs, whereas -qcov hsp

232 perc 50 was set for the rest.

$233 \quad 3.2$ blast filteripynb filters BLASTn results by using parameters according to Table 1.

234 First, each file is filtered by a length range defined by min len and max len. Afterwards, a

235 length threshold range is calculated by multiplying the query length by a min and max

236 subject length subject length. The subject sequence has to be inside this range to be

237 considered valid. Later, minimum identity percentage and query coverage are required

238 for the sequences to be valid. Finally, duplicated hits are removed by searching those

239 whose start and end positions overlaps within a margin of plus or minus $5 \mathrm{nt}$.

2404.1 annotate.ipynb transforms the BLAST tab-delimited results to a gff3 format file, 241 adding a detailed description for each TE. The description includes TE id (a numeric 242 identifier of the element after clustering) source name (original id name of the element 243 before clustering) type (family type of the element), source (program or tool from which 244 the TEs were detected) and unique id (unique identifier for copy element).

\section{LTR age}

To determine tentative LTRs insertion age we used tephra Itrage command implemented by the Tephra package. This command uses the Tephra-discovered full length LTRs, aligns the LTR sequences and generates a neighbor-joining guide tree with MUSCLE (Edgar 2004). The alignment and guide tree are used to generate an alignment in PHYLIP format. A likelihood divergence estimate was calculated with baseml from PAML (Yang 2007) by specifying the K80 substitution model. This divergence value (hereafter d) was used to calculate LTR-RT age with the formula $T=$ $\mathrm{d} / 2 \mathrm{r}$, where $\mathrm{r}=1 \mathrm{e} 8 \mathrm{is}$ the default substitution rate.

\section{Data resource}

Scripts from this work, including all pipeline steps as well as circos ideogram, distance histogram and LTR age plot scripts are available at (https://github.com/DiegoZavallo/TE Discovery). Annotation and fasta files are available as supporting information.

\section{Results and Discussion}

The goal of the present work was to assemble a comprehensive TE repertoire of the potato genome and provide legible, ready-to-use files for the scientific community. To 
268 address this issue, we used a combination of different approaches to identify TEs such

269 as similarity-based, structure-based and mapping-based strategies. Moreover, we 270 introduce a set of scripts to gather, detect, filter and ultimately annotate TE copies from 271 all classes across the potato genome and present gff3 files of TE features. Additionally, 272 we display the accumulation and distribution across the genome of the different types of 273 TEs and a table summarizing data and metrics, such as distances to nearest gene and 274 LTRs insertion ages.

275

\section{TEs in the potato genome}

Public S. tuberosum Repbase library (Jurka et al., 2005), the potato reference genome and Illumina small RNA-seq data were used as input data. TEs family detection was executed by applying two "All TEs" tools: Tephra and TASR, which discover de novo TEs with structure-based and map-based approaches, respectively. To complement the search, we applied "Specific TEs" tools: MITE Tracker and SINE_Scan. The obtained sequences were merged into multi-fasta files, one for each type of TE, and headers were renamed. A clustering step was carried out with Vsearch to reduce redundancy (Online Resource 1). Next, detection of copies in the genome of the different TE families was achieved by conducting a BLAST search with specific parameters according to the type of TE (see Materials and Methods section). A copy filter step was implemented by establishing several rules with very astringent criteria to detect "potential autonomous TEs" and a second set of rules for "All TEs" with more relaxed parameters to account for autonomous and non-autonomous TEs of all types (Table 1). Finally, an annotation step was performed to generate gff3 files containing a description for each element that includes TE type and the detection tool that identified it. Figure 1 shows an overview of the pipeline used to detect and re-annotate the potato TEs.

TE content is highly variable in plants and usually displays a positive correlation with genome size. For instance, as much as $85 \%$ of maize genome or $70 \%$ of Norway spruce genome (Nystedt et al., 2013) has been annotated as transposons including unclassified ones, whereas in the more compact Arabidopsis thaliana genome TE content is only $21 \%$ (Ahmed et al., 2011). In potato, the data presented by Mehra et al. (2015) comprised an annotation file of 1,061,377 repetitive elements, including rRNA, tRNA, simple repeats and low complexity elements which represents almost $50 \%$ of the genome. When only the most complex elements (i.e. transposons) were taking into account, the coverage percentage dropped to nearly $34 \%$. 
However, our pipeline revealed a TE content of $\sim 16 \%$ (excluding the unanchored ChrUn), representing half of the genomic coverage according to the data presented by Mehra et al. (2015).

Of those $16 \%$, LTRs comprised around $13 \%$ of the potato genome, which corresponds to over $80 \%$ of the total TEs. The most abundant superfamily was Gypsy, whereas the other types of TEs barely made up $1 \%$ of the genome coverage. For instance, each DNA TE (TIRs, MITEs and Helitrons) covered almost the same percentage of the genome with $0.51,0.72$ and $0.72 \%$, respectively. These coverage ratio patterns are in agreement with results from most plant genomes that have TE identification projects (Du et al., 2010; Andorf et al., 2016; Badouin et al., 2017; Alaux et al., 2018). Table 2 summarizes the amount and diversity of all identified TEs, filtered copies and proportion in the genome of all TE families.

To understand more deeply the discrepancy between the coverage of the TE genome presented here and the one reported by Mehra et al. (2015), we should observe other differences between both works. For instance, even though we describe all types of TE families reported by Wicker et al. (2007), including LARDs, TRIMs and MITEs, which were absent in Mehra study, we annotated less than half of the sequences. We applied a clustering method to decrease redundancy and established-a set of filters selected specifically for each type of TEs, which, is aimed to reduce false signal. Moreover, as already mentioned, Mehra et al. used a unique tool that relies on repetitiveness-based strategy, leaving aside a wide variety of detection methods that in this work we have combined, which is indicated to improve TE detection efficiency (Kamoun et al., 2013; Hoen et al., 2015; Arensburger et al., 2016).

We scored a total of 243,010 elements compared to 629,713 complex elements previously found by Mehra et. al. and when compared, 198,025 (81\%) of our sequences overlapped with their set, which evidences the effectiveness of both annotation pipelines.

To test the effectiveness of the pipeline, we run it on a well annotated genome such as soybean. SoyTEdb represents an example of a thoroughly annotated TE database in which a combination of structure-based and homology-based approaches was used to structurally annotate and clearly categorize TEs in the soybean genome (Du et al., 2010). The authors reported over 38,000 TEs representing $\sim 17 \%$ of the genome. However, when they informed the genome coverage they included fragments defined by RepeatMasker (i.e. low complex repeats) rising up to $58 \%$ of the soybean genome. These data may indicate the existence of a large set of repetitive sequences in the genome that cannot be annotated as TE with current knowledge about the structure of TE. One hypothesis that could arise from this is that the structural patterns that 
341 represent these non-annotated TEs are not yet thoroughly described, or they are just

342 repetitive DNA that simply cannot be assigned as TEs. We used the 38,000 annotated

343 TEs to run our pipeline for copy elements discovery and filtering steps and we came out

344 with similar genomic coverage ( 23\%) and more than $75 \%$ of the TEs sequences

345 overlapped. Even though this was only a test, since the full run of our pipeline on

346 another species would require a more comprehensive approach which exceeds this

347 work, it validates that this pipeline does not underestimate the occurrence of TEs in the 348 genome.

349 Finally, we deliver ready-to-use annotation files in gff3 format of all TEs annotated 350 with our pipeline with detailed descriptions in the ninth column including TE id, source name, type, source and unique id (Online Resource 2).

352

\section{Distribution of TEs across the potato genome}

It is well know that the distribution, amount and genome coverage of TEs vary greatly, particularly between plants and animals, where LTRs and non-LTRs (LINEs and SINEs) are the predominant type of TE, respectively (Chalopin et al. 2015). Moreover, TE distribution is highly dependent on the family type. Some TEs are more prone to concentrate in regions near protein coding genes while others are more equally distributed along the genome.

To assess the type-specific landscape of the diverse TE categories, we performed circos ideograms for each TE type separated by class, as well as gene density as reference. Each concentric circle represents the coverage percentage of one type of TE. Since they have different coverage ranges, each type has its own color pallet to appreciate the distribution across the chromosomes (Figure 2).

Left panel of Figure 2 shows the class I elements, where LTRs stand out, not only for their clear pattern of clustering around centromeric and pericentromeric regions, but also for their high coverage in some areas of the chromosomes. For instance, each dark red line of the second circle represent up to $36 \%$ of LTRs coverage per Mb, which explains the $13 \%$ genome-wide coverage for this kind of TE (Table 2). Furthermore, LTRs distribution is virtually opposite to protein coding gene distribution (Figure 2, left panel). This behavior has already been reported for other plants (Baucom et al., 2009; Paterson et al., 2009; Badouin et al., 2017).

Conversely, SINEs, which are the least represented type (besides LARDs which are not shown since we discovered only 18 elements) seem to have an even distribution pattern with a slight tendency towards telomeric and subtelomeric regions in some chromosomes. LINEs and TRIMs also display homogeneous distribution patterns with 
some coverage hotspots, mainly near telomeric regions. Due to their length and filtering parameters established, only 248 LINEs were found in the genome using our methodology, contrasting with more than 50,000 elements found by Mehra et al. (2015). Given that Heitkam et al. (2014) extracted 59,390 intact LINE sequences from 23 plant genomes in order to classify them into families, it is somehow unlikely that potato alone could have 50,000 LINEs. A look at the data presented by Mehra et al. (2015) shows that some elements annotated as LINEs are small fragments with some identity to LINEs given by the RepeatMasker tool which in our case were removed by the filtering process.

In a recent work, Gao et al. (2016) performed a comprehensive analysis of TRIMs in 48 plant genomes, including $S$. tuberosum. They observed that TRIMs are generally enriched in genic regions and likely play a role in gene evolution (Gao et al., 2016). They discovered 12,473 copies in the potato genome representing $0.46 \%$ of the genome, which is consistent with our results (Table 2).

Right panel of Figure 2 shows class II TEs distribution in which MITEs display a clear concentration pattern around gene-rich subtelomeric regions. According to previous works, MITEs are often found close to or within genes, where they affect gene expression (Bureau and Wessler, 1994b). Indeed, MITEs may affect gene regulation via small RNA pathways, in addition to play the canonical role of TEs in the evolution by altering gene structure (Kuang et al., 2009; Gagliardi et al., 2019).

TIRs and Helitrons displayed an unbiased distribution across the chromosomes (Figure 2, right panel). Helitron chromosome distribution seems to vary by species. While in Arabidopsis Helitrons are enriched in gene-poor pericentromeric region, in maize they are more abundant in gene-rich regions (Yang and Bennetzen, 2009). Rice, on the other hand, exhibited a more erratic pattern of Helitron distribution (Yang and Bennetzen, 2009), more similar to our results.

In sum, this kind of analysis allowed us to have a holistic view of the different distribution patterns of TEs across the genome and to elucidate their potential role in transcriptional gene regulation.

\section{TEs and genes}

The importance of TEs accumulation near genes, where these elements could influence gene expression, has been extensively reported (Bureau and Wessler 1994b,a; Wang et al., 2013). As we described above, we found a strong positive correlation between LTRs and pericentromeric regions as well as a positive correlation between MITEs and rich-gene subtelomeric regions. Hence, we determined the 
415 distances from the different types of elements to the closest gene in the genome in

416 order to assess how many TEs are likely within or close to genes. For this purpose, we

417 computed metrics such as median distance to the closest gene and percentage of TEs

418 overlapping gene transcripts or near coding regions. LTRs were found to be generally

419 far from genes with a median distance of $10.25 \mathrm{~kb} ; 9.01 \%$ overlap with coding sequence

420 genes and only $5.11 \%$ are in the immediate vicinity (up to $1 \mathrm{~kb}$ from the nearest gene),

421 totaling a $32.23 \%$ within the first $5 \mathrm{~kb}$, including elements within transcripts (Figure 3 and

422 Online Resource 3). TRIMs exhibit a similar behavior, with a median distance of $8.12 \mathrm{~kb}$,

$4236.43 \%$ elements located within a gene and $6.19 \%$ in the range of $0-1 \mathrm{~kb}$ to the nearest

424 transcript, comprising a $36.62 \%$ in the first $5 \mathrm{~kb}$. In contrast, LINEs and SINEs are close

425 to genes (median distance of $4.16 \mathrm{~kb}$ and $3.86 \mathrm{~kb}$, respectively) with more than $20 \%$

426 elements within a transcript and rising up to $>50 \%$ in the $0-5 \mathrm{~Kb}$ range $(52.44 \%$ and

$42756.20 \%$ respectively).

428 Class II TEs also have more than $50 \%$ of their elements within the first $5 \mathrm{~kb}$.

429 However, TIRs and MITEs distribution differs from that of Helitrons. TIRs and MITEs are

430 the only types of TEs that appear to have less elements within a gene than in the $0-1 \mathrm{~kb}$

431 range (TIRs: $10.05 \%$ against $15.5 \%$ and a median distance of $3.32 \mathrm{~kb}$; MITEs: $7.07 \%$

432 against $14.21 \%$ and a median distance of $3.53 \mathrm{~kb}$ ). On the other hand, Helitrons display

433 a similar pattern to LINEs and SINEs with a median of $4.56 \mathrm{~kb}$ and $23.65 \%$ elements

434 within a transcript. These differences can be observed in the less pronounced curve of

435 TIR and MITE histograms demonstrating that a significant proportion of elements are

436 indeed in the proximity of genes but not necessarily within them. (Figure 3, right panel

437 and Online Resource 3).

438 Overall, DNA TEs and nonLTRs retrotransposons have more than $50 \%$ of the 439 elements inserted within transcripts or in a range of $0-5 \mathrm{~kb}$ from the nearest gene.

440 Several studies have reported examples of transcriptional impact due to TEs insertion 441 near genes in tomato (Xiao et al., 2008; Quadrana et al., 2014), potato (Momose et al., 442 2010; Kloosterman et al., 2013), melon (Martin et al., 2009) and orange (Butelli et al., 443 2012) among others. Several mechanisms including disruption of promoter or reduction 444 of transcription through the spread of epigenetic silencing often suppress expression. 445 However, TE can also introduce new sequences in the promoter, leading to up446 regulation of proximal gene (Yan et al., 2004; Cowley and Oakey, 2013; Dubin et al., 447 2018). Insertion of a TE into the coding sequence can disrupt gene function, generally 448 resulting in loss-of-function mutations, particularly if located in an exon. Intronic TEs can 449 also be harmful, for instance by altering splicing patterns (Saze et al., 2013; Ong450 Abdullah et al., 2015). 
In contrast, LTRs and TRIMs located near genes barely exceed $30 \%$ and, with a few exceptions, they appear in intergenic, heterochromatic and gene-poor-regions (Kumar and Bennetzen, 1999). In Arabidopsis, Wang et al. (2013) reported that gene expression is positively correlated with the distance of the gene to the nearest TE, and negatively correlated with the number of proximal TEs. Whether LTR-like TEs specifically target these regions or if they are simply not selected against and accumulated in regions nearby genes remains unclear (Sigman and Slotkin, 2016).

\section{LTR elements age and evolution}

LTRs are the most represented TEs in the majority of plant genomes, encompassing more than $75 \%$ of the nuclear genome of some species (Kumar and Bennetzen, 1999; Paz et al., 2017). For this reason, we decided to explore the evolutionary history of LTRs during potato genome evolution.

We analyzed the age and distribution of the elements discovered by Tephra (Staton, 2018) by means of tephra Itrage command that allowed the characterization of phylogenetic substructure within families of LTR retrotransposons. A total of 8,034 fulllength LTRs were analyzed in terms of chromosomal distribution and insertion age by plotting all LTR elements together (Figure 4, upper panel), or grouped into superfamiles (Figure 4, bottom panel).

Younger LTRs are enriched in euchromatic subtelomeric regions and correspond mainly to Copia (RLC) family of TEs (average insertion age of 2.54 mya), whereas older LTRs are more abundant in heterochromatic pericentromeric regions where Gypsy (RLG) elements are mostly located (average insertion age of 4.14 mya). These distributions are in agreement with previous findings in maize (Sun et al., 2018), wheat (Luo et al., 2017) and tomato (Paz et al., 2017).

Unclassified LTRs $(R L X)$ display a more even distribution, although slightly towards to pericentromeric regions, as well as an intermediate insertion age (average insertion age of 3.78 mya) (Figure 4, bottom panel).

To determine whether these aging differences were a family trait or a genomic regiondependent mutation/substitution rate, we divided each chromosome in euchromatic and heterochromatic regions. In order to do so, we compared the pachytene karyotype previously published (Consortium et al., 2011) with the chromosomal ideograms we produced, and plotted the frequency of each LTR family by insertion age according to the karyotype determined region (Figure 5, upper panel). Gypsy family (RLG) display a Gaussian distribution centered around four millions years both in euchromatic and heterochromatic regions, whereas Copia family $(R L C)$ have a chi-square distribution 
488 with a peak between two and three millions years on both regions. These results

489 suggest that the insertion age of LTRs retrotransposons depends on the superfamily

490 and not on differential mutation/substitution rate by region.

491 Furthermore, we plotted ten random independent families encompassing different member sizes from RLG, RLC and RLX to assess the age distribution of individual elements by region (Figure 5, bottom panel). Only four out of the ten evaluated Gypsy families showed significant differences (t-test $p<0.05$ ) in aging by region. In all the cases, heterochromatic elements were older than euchromatic elements, which reflect a slight age difference within elements of the same family owing to their insertion sites. This suggests that insertions into heterochromatic regions are more likely to persist for longer periods of time.

Overall, these variances may have a component based on differential mutation/substitution rate by region but more importantly it is strongly affected by superfamily traits. A recent study by Quadrana et al. (2019) described the mechanisms for which several TEs of the Copia superfamily preferentially integrate within genes by association with H2A.Z-containing nucleosomes. Moreover, they suggest that the role of H2A.Z in the integration of Copia retrotransoposons has been evolutionary conserved since the last common ancestor of plants and fungi (Quadrana et al., 2019).

On the other hand, Gypsy superfamily harbors a chromodomain that interacts with repressive histone marks such as $\mathrm{H} 3 \mathrm{~K} 9 \mathrm{~m} 2$ which targets to heterochromoatin (Sultana

\section{Conclusion}

TEs have been historically neglected in genome assembly projects, partially due to their repetitive nature but also because their heterogeneity in sequence, size, number of copies, distribution and mutation rates both inter and intra species, make them very difficult to detect accurately (Bourque et al., 2018).

However, in the past years, TEs discovery and annotation for the main crops have emerged with diverse results of coverage, complexity and accuracy. For instance, by using the CLARITE software on the IWGSC RefSeq v1.0 genome assembly, Alaux et al. (2018) found over 5 million elements from all types of TEs in wheat.

The Rice TE Database collects repeat sequences and TEs of several species of Oryza (rice) genus. All sequences have been characterized adopting Wicker's classification code and extending it by encoding new TE superfamilies and non-TE repeats. Particular emphasis was given to the proper classification of sequences and to the removal of nested insertions (Copetti et al., 2015). 
As we described above, potato has some TE sequences annotated on the RepBase, essentially, a list based on RepeatMasker provided by the Potato Genome Sequencing Consortium and the work from Mehra et al. (2015), which is based on RepeatModeler. However, this worldwide important crop still lacked a comprehensive, multiple-based discovery approach focused on transposon elements annotation.

This work arises as a need of a reliable potato TE atlas for ongoing projects that involve epigenetic and transcriptional regulation for different Solanum tuberosum in contrasting environments.

Plants are known for their phenotypic plasticity and good adaptation to environmental changes due to their sessile condition. There is an increasing evidence of the impact that TEs have on the transcriptome on response to stress. For example, TEs may have direct effects on genes regulation, by providing them new coding or regulatory sequences, changes on the epigenetic status of the chromatin close to genes, and more subtle effects by imposing diverse evolutionary constraints to different chromosomal regions (Makarevitch et al., 2015; Vicient and Casacuberta, 2017; Cambiagno et al., 2018). Finding common patterns or specific alterations on these features (TEs) and linking them with sRNAs profiles and DNA methylation patterns could help researchers to elucidate the underlying mechanisms of transcriptional changes during different stress conditions.

As mentioned above, the current data regarding TEs on potato genome is either scare or imprecise for transcriptional analysis. Given the importance of transposon elements, we provide here a comprehensive potato TE landscape, based on a wide variety of identification tools and approaches, clustering methods, copies detection, filtering rules and clear outputs, that the scientific community will likely use for metadata analysis.

\section{Acknowledgements}

The authors would like to thank Dr. Soledad Lucero and Dr. Julia Sabio y Garcia for the assistance with English-language editing, and Humberto Julio Debat for his critical comments on the manuscript. This work was supported by the Instituto Nacional de Tecnología Agropecuaria (INTA) and by ANPCyT PICT 2015-1532, and PICT 20160429. The funders had no role in this study design, data collection and analysis, decision to publish or preparation of the manuscript.

\section{Author contributions}


562 DZ: Conceptualization, Data curation, Formal analysis, Investigation, Methodology,

563 Visualization, Writing - original draft, Writing - review \& editing. JMC: Formal analysis,

564 Data curation, Investigation, Methodology, Software, Writing - review \& editing.

565 MG: Investigation, Writing - review \& editing. ML: Investigation, Writing - review \&

566 editing. LSV: Formal analysis, Investigation, Writing - review \& editing. RWM: Funding

567 acquisition, Investigation, Writing - review \& editing. SA: Conceptualization, Funding

568 acquisition, Investigation, Project administration, Resources, Supervision, Writing -

569 review \& editing.

570

571

572

573

\section{Conflict of interest}

The authors have no conflicts of interest to declare

574

575

576

577

578

579

580

581

582

583

584

585

586

587

588

589

590

591

592

593

594

595

596

597

598

599

600

\section{Electronic Supplementary Material}

Online Resource 1. Fasta files containing family sequences for each type transposon elements.

Online Resource 2. gff3 file containing all TEs copy coordinates including Chr00.

Online Resource 3. Table resuming metrics of TEs to the nearest genes.

4

6




\section{References}

Ahmed, I., Sarazin, A., Bowler, C., Colot, V., and Quesneville, H. (2011). Genome-wide evidence for local DNA methylation spreading from small RNA-targeted sequences in Arabidopsis. Nucleic Acids Research, 39(16):6919-6931.

Alaux, M., Rogers, J., Letellier, T., Flores, R., Alfama, F., Pommier, C., Mohellibi, N., Durand, S., Kimmel, E., Michotey, C., et al. (2018). Linking the International Wheat Genome Sequencing Consortium bread wheat reference genome sequence to wheat genetic and phenomic data. Genome biology, 19(1):111.

Andorf, C. M., Cannon, E. K., Portwood, J. L., Gardiner, J. M., Harper, L. C., Schaeffer, M. L., Braun, B. L., Campbell, D. A., Vinnakota, A. G., Sribalusu, V. V., et al. (2016). MaizeGDB update: new tools, data and interface for the maize model organism database. Nucleic Acids Research, 44(D1):D1195-D1201.

Appels, R., Eversole, K., Feuillet, C., Keller, B., Rogers, J., Stein, N., Pozniak, C. J., Choulet, F., Distelfeld, A., Poland, J., et al. (2018). Shifting the limits in wheat research and breeding using a fully annotated reference genome. Science, 361(6403):eaar7191.

Arensburger, P., Piégu, B., and Bigot, Y. (2016). The future of transposable element annotation and their classification in the light of functional genomics - what we can learn from the fables of Jean de la Fontaine? Mobile Genetic Elements, 6(6):e1256852.

Badouin, H., Gouzy, J., Grassa, C. J., Murat, F., Staton, S. E., Cottret, L., LelandaisBriére, C., Owens, G. L., Carrére, S., Mayjonade, B., et al. (2017). The sunflower genome provides insights into oil metabolism, flowering and Asterid evolution. Nature, 546(7656):148.

Baucom, R. S., Estill, J. C., Chaparro, C., Upshaw, N., Jogi, A., Deragon, J.-M., Westerman, R. P., SanMiguel, P. J., and Bennetzen, J. L. (2009). Exceptional diversity, non-random distribution, and rapid evolution of retroelements in the B73 maize genome. PLoS Genetics, 5(11):e1000732.

Berthelier, J., Casse, N., Daccord, N., Jamilloux, V., Saint-Jean, B., and Carrier, G. (2018). A transposable element annotation pipeline and expression analysis reveal potentially active elements in the microalga Tisochrysis lutea. BMC Genomics, 19(1):378.

Bourque, G., Burns, K. H., Gehring, M., Gorbunova, V., Seluanov, A., Hammell, M., Imbeault, M., Izsvak, Z., Levin, H. L., Macfarlan, T. S., et al. (2018). Ten things you should know about transposable elements. Genome Biology, 19(1):199.

Bureau, T. E. and Wessler, S. R. (1994a). Mobile inverted-repeat elements of the tourist family are associated with the genes of many cereal grasses. Proceedings of the National Academy of Sciences, 91(4):1411-1415.

Bureau, T. E. and Wessler, S. R. (1994b). Stowaway: a new family of inverted repeat elements associated with the genes of both monocotyledonous and dicotyledonous plants. The Plant Cell, 6(6):907-916. 
Butelli, E., Licciardello, C., Zhang, Y., Liu, J., Mackay, S., Bailey, P., Reforgiato-Recupero, G., and Martin, C. (2012). Retrotransposons control fruit-specific, cold-dependent accumulation of anthocyanins in blood oranges. The Plant Cell, 24(3):1242-1255.

Cambiagno, D. A., Nota, F., Zavallo, D., Rius, S., Casati, P., Asurmendi, S., and Alvarez, M. E. (2018). Immune receptor genes and pericentromeric transposons as targets of common epigenetic regulatory elements. The Plant Journal, 96(6):1178-1190.

Chalopin, D., Naville, M., Plard, F., Galiana, D., and Volff, J.-N. (2015). Comparative analysis of transposable elements highlights mobilome diversity and evolution in vertebrates. Genome Biology and Evolution, 7(2):567-580.

Consortium, P. G. S. et al. (2011). Genome sequence and analysis of the tuber crop potato. Nature, 475(7355):189.

Copetti, D., Zhang, J., El Baidouri, M., Gao, D., Wang, J., Barghini, E., Cossu, R.M., Angelova, A., Maldonado, L. CE., Roffler, S., Ohyanagi, H., Wicker, T., Fan, C., Zuccolo, A., Chen, M., Costa de Oliveria, A., Han, B..., Henry, R., Hsing, Y-I., Kurata, N., Wang, W., Jackson, S.A., Panaud, O., Wing, R.A. (2015). RiTE database: a resource database for genus-wide rice genomics and evolutionary biology. BMC Genomics, 16:538

Cowley, M. and Oakey, R. J. (2013). Transposable elements re-wire and fine-tune the transcriptome. PLoS Genetics, 9(1):e1003234.

Crescente, J. M., Zavallo, D., Helguera, M., and Vanzetti, L. S. (2018). Mite tracker: an accurate approach to identify miniature inverted-repeat transposable elements in large genomes. BMC Bioinformatics, 19(1):348.

Devaux, A., Kromann, P., and Ortiz, O. (2014). Potatoes for sustainable global food security. Potato Research, 57(3-4):185-199.

Du, J., Grant, D., Tian, Z., Nelson, R. T., Zhu, L., Shoemaker, R. C., and Ma, J. (2010). SoyTEdb: a comprehensive database of transposable elements in the soybean genome. BMC Genomics, 11(1):113.

Dubin, M. J., Scheid, O. M., and Becker, C. (2018). Transposons: a blessing curse. Current Opinion in Plant Biology, 42:23-29.

Edgar, R. C. (2004). MUSCLE: multiple sequence alignment with high accuracy and high throughput. Nucleic Acids Research, 32(5):1792-1797.

El Baidouri, M., Kim, K. D., Abernathy, B., Arikit, S., Maumus, F., Panaud, O., Meyers, B. C., and Jackson, S. A. (2015). A new approach for annotation of transposable elements using small rna mapping. Nucleic Acids Research, 43(13):e84-e84.

Ellinghaus, D., Kurtz, S., and Willhoeft, U. (2008). LTRharvest, an efficient and flexible software for de novo detection of LTR retrotransposons. BMC Bioinformatics, 9(1):18.

Gagliardi, D., Cambiagno, D. A., Arce, A. L., Tomassi, A. H., Giacomelli, J. I., Ariel, F. D., Manavella, P. A. (2019). Dynamic regulation of chromatin topology and transcription by inverted repeat-derived small RNAs in sunflower. Proceedings of the National Academy of Sciences, 116 (35): 17578-1758. 
Gao, D., Li, Y., Do Kim, K., Abernathy, B., and Jackson, S. A. (2016). Landscape and evolutionary dynamics of terminal repeat retrotransposons in miniature in plant genomes. Genome Biology, 17(1):7.

Gremme, G., Steinbiss, S., and Kurtz, S. (2013). Genometools: a comprehensive software library for efficient processing of structured genome annotations. IEEE/ACM Transactions on Computational Biology and Bioinformatics (TCBB), 10(3):645-656.

Heitkam, T., Holtgrawe, D., Dohm, J. C., Minoche, A. E., Himmelbauer, H., Weisshaar, B., and Schmidt, T. (2014). Profiling of extensively diversified plant lines reveals distinct plant-specific subclades. The Plant Journal, 79(3):385-397.

Hirsch, C. D. and Springer, N. M. (2017). Transposable element influences on gene expression in plants. Biochimica et Biophysica Acta (BBA)-Gene Regulatory Mechanisms, 1860(1):157-165.

Hoede, C., Arnoux, S., Moisset, M., Chaumier, T., Inizan, O., Jamilloux, V., and Quesneville, H. (2014). PASTEC: an automatic transposable element classification tool. PloS One, 9(5):e91929.

Hoen, D. R., Hickey, G., Bourque, G., Casacuberta, J., Cordaux, R., Feschotte, C., FistonLavier, A.-S., Hua-Van, A., Hubley, R., Kapusta, A., et al. (2015). A call for benchmarking transposable element annotation methods. Mobile DNA, 6(1):13.

Hosaka, A. and Kakutani, T. (2018). Transposable elements, genome evolution and transgenerational epigenetic variation. Current Opinion in Genetics \& Development, $49: 43-48$.

Judd, J. and Feschotte, C. (2018). Gene expression: Transposons take remote control. eLife, 7:e40921.

Jurka, J., Kapitonov, V. V., Pavlicek, A., Klonowski, P., Kohany, O., and Walichiewicz, J. (2005). Repbase update, a database of eukaryotic repetitive elements. Cytogenetic and Genome Research, 110(1-4):462-467.

Kamoun, C., Payen, T., Hua-Van, A., and Filée, J. (2013). Improving prokaryotic transposable elements identification using a combination of de novo and profile $\mathrm{hmm}$ methods. BMC Genomics, 14(1):700.

Kloosterman, B., Abelenda, J. A., Gomez, M. d. M. C., Oortwijn, M., de Boer, J. M., Kowitwanich, K., Horvath, B. M., van Eck, H. J., Smaczniak, C., Prat, S., et al. (2013). Naturally occurring allele diversity allows potato cultivation in northern latitudes. Nature, 495(7440):246.

Kuang, H., Padmanabhan, C., Li, F., Kamei, A., Bhaskar, P. B., Ouyang, S., Jiang, J., Buell, C. R., and Baker, B. (2009). Identification of miniature inverted-repeat transposable elements (MITEs) and biogenesis of their siRNAs in the Solanaceae: new functional implications for MITEs. Genome Research, 19(1):42-56.

Kumar, A. and Bennetzen, J. L. (1999). Plant retrotransposons. Annual Review of Genetics, 33(1):479-532.

Luo, M.-C., Gu, Y. Q., Puiu, D., Wang, H., Twardziok, S. O., Deal, K. R., Huo, N., Zhu, T., Wang, L., Wang, Y., et al. (2017). Genome sequence of the progenitor of the wheat D genome aegilops tauschii. Nature, 551(7681):498. 
Macas, J., Meszaros, T., and Nouzova, M. (2002). PlantSat: a specialized database for plant satellite repeats. Bioinformatics, 18(1):28-35.

Makarevitch, I., Waters, A. J., West, P. T., Stitzer, M., Hirsch, C. N., Ross-Ibarra, J., and Springer, N. M. (2015). Transposable elements contribute to activation of maize genes in response to abiotic stress. PLoS Genetics, 11(1):e1004915.

Mao, H. and Wang, H. (2016). Sine_scan: an_efficient tool to discover short interspersed nuclear elements (SINEs) in large-scale genomic datasets. Bioinformatics, 33(5):743745.

Martin, A., Troadec, C., Boualem, A., Rajab, M., Fernandez, R., Morin, H., Pitrat, M., Dogimont, C., and Bendahmane, A. (2009). A transposon-induced epigenetic change leads to sex determination in melon. Nature, 461(7267):1135.

McCarthy, E. M. and McDonald, J. F. (2003). LTR_STRUC: a novel search and identification program for LTR retrotransposons. Bioinformatics, 19(3):362-367.

Mehra, M., Gangwar, I., and Shankar, R. (2015). A deluge of complex repeats: the Solanum genome. PloS One, 10(8):e0133962.

Momose, M., Abe, Y., and Ozeki, Y. (2010). Miniature inverted-repeat transposable elements of stowaway are active in potato. Genetics, 186(1):59-66.

Nussbaumer, T., Martis, M. M., Roessner, S. K., Pfeifer, M., Bader, K. C., Sharma, S., Gundlach, H., and Spannagl, M. (2012). MIPS PlantsDB: a database framework for comparative plant genome research. Nucleic Acids Research, 41(D1):D1144-D1151.

Nystedt, B., Street, N. R., Wetterbom, A., Zuccolo, A., Lin, Y.-C., Scofield, D. G., Vezzi, F., Delhomme, N., Giacomello, S., Alexeyenko, A., et al. (2013). The Norway spruce genome sequence and conifer genome evolution. Nature, 497(7451):579.

Ong-Abdullah, M., Ordway, J. M., Jiang, N., Ooi, S.-E., Kok, S.-Y., Sarpan, N., Azimi, N., Hashim, A. T., Ishak, Z., Rosli, S. K., et al. (2015). Loss of Karma transposon methylation underlies the mantled somaclonal variant of oil palm. Nature, 525(7570):533.

Ouyang, S. and Buell, C. R. (2004). The TIGR Plant Repeat Databases: a collective resource for the identification of repetitive sequences in plants. Nucleic Acids Research, 32(suppl 1):D360-D363.

Paterson, A. H., Bowers, J. E., Bruggmann, R., Dubchak, I., Grimwood, J., Gundlach, H., Haberer, G., Hellsten, U., Mitros, T., Poliakov, A., et al. (2009). The Sorghum bicolor genome and the diversification of grasses. Nature, 457(7229):551.

Paz, R. C., Kozaczek, M. E., Rosli, H. G., Andino, N. P., and Sanchez-Puerta, M. V. (2017). Diversity, distribution and dynamics of full-length Copia and Gypsy LTR retroelements in Solanum lycopersicum. Genetica, 145(4-5):417-430.

Platt, R. N., Blanco-Berdugo, L., and Ray, D. A. (2016). Accurate transposable element annotation is vital when analyzing new genome assemblies. Genome Biology and Evolution, 8(2):403-410. 
Quadrana, L., Almeida, J., Asís, R., Duffy, T., Dominguez, P. G., Bermúdez, L., Conti, G., Da Silva, J. V. C., Peralta, I. E., Colot, V., et al. (2014). Natural occurring epialleles determine vitamin $E$ accumulation in tomato fruits. Nature Communications, 5:4027.

Quadrana, L., Etcheverry, M., Gilly, A., Caillieux, E., Madoui, M. A., Guy, J., \& Aury, J. M. (2019). Transposition favors the generation of large effect mutations that may facilitate rapid adaptation. Nature Communications, 10(1), 3421.

Roessler, K., Bousios, A., Meca, E., and Gaut, B. S. (2018). Modeling interactions between transposable elements and the plant epigenetic response: a surprising reliance on element retention. Genome Biology and Evolution, 10(3):803-815.

Saze, H., Kitayama, J., Takashima, K., Miura, S., Harukawa, Y., Ito, T., and Kakutani, T. (2013). Mechanism for full-length RNA processing of Arabidopsis genes containing intragenic heterochromatin. Nature Communications, 4:2301.

Schnable, P. S., Ware, D., Fulton, R. S., Stein, J. C., Wei, F., Pasternak, S., Liang, C., Zhang, J., Fulton, L., Graves, T. A., et al. (2009). The B73 maize genome: complexity, diversity, and dynamics. Science, 326(5956):1112-1115.

Sigman, M. J. and Slotkin, R. K. (2016). The first rule of plant transposable element silencing: location, location, location. The Plant Cell, 28(2):304-313.

Smit, A. F., Hubley, R., and Green, P. (1996). Repeatmasker.

Staton, E. (2018). Tephra a tool for discovering transposable elements and describing patterns of genome evolution.

Sultana, T., Zamborlini, A., Cristofari, G., \& Lesage, P. (2017). Integration site selection by retroviruses and transposable elements in eukaryotes. Nature Reviews Genetics, 18(5), 292.

Sun, S., Zhou, Y., Chen, J., Shi, J., Zhao, H., Zhao, H., Song, W., Zhang, M., Cui, Y., Dong, $X$., et al. (2018). Extensive intraspecific gene order and gene structural variations between Mo17 and other maize genomes. Nature Genetics, 50(9):1289.

Vicient, C. M. and Casacuberta, J. M. (2017). Impact of transposable elements on polyploid plant genomes. Annals of Botany, 120(2):195-207.

Wang, X., Weigel, D., and Smith, L. M. (2013). Transposon variants and their effects on gene expression in Arabidopsis. PLoS Genetics, 9(2):e1003255.

Wicker, T., Matthews, D. E., and Keller, B. (2002). TREP: a database for Triticeae repetitive elements. Trends in Plant Science, 7(12):561-562.

Wicker, T., Sabot, F., Hua-Van, A., Bennetzen, J. L., Capy, P., Chalhoub, B., Flavell, A., Leroy, P., Morgante, M., Panaud, O., et al. (2007). A unified classification system for eukaryotic transposable elements. Nature Reviews Genetics, 8(12):973.

Xiao, H., Jiang, N., Schaffner, E., Stockinger, E. J., and Van Der Knaap, E. (2008). A retrotransposon-mediated gene duplication underlies morphological variation of tomato fruit. Science, 319(5869):1527-1530. 
Xiong, W., He, L., Lai, J., Dooner, H. K., and Du, C. (2014). HelitronScanner uncovers a large overlooked cache of Helitron transposons in many plant genomes. Proceedings of the National Academy of Sciences, page 201410068.

Yang, L. and Bennetzen, J. L. (2009). Distribution, diversity, evolution, and survival of Helitrons in the maize genome. Proceedings of the National Academy of Sciences, 106(47):19922-19927.

Yang, Z. (2007). PAML 4: phylogenetic analysis by maximum likelihood. Molecular Biology and Evolution, 24(8):1586-1591.

Table 1: TEs filtering parameters. Parameters established to filter copies element for "Autonomous TEs" and "All TEs" which includes autonomous and non-autonomous elements. min-len: minimum element length; max-len: maximum element length; minpid: minimum query identity percentage; min-threshold: minimum threshold length between query and subject; max-threshold: maximum threshold length between query and subject; min-cov: minimum query coverage percentage; overlap: margin of plus or minus nucleotides overlap between query and subject to be considered as duplicates.

\begin{tabular}{lccccccc}
\hline Family & $\begin{array}{c}\text { min-len } \\
\text { (nt) }\end{array}$ & $\begin{array}{c}\text { max- } \\
\text { len (nt) }\end{array}$ & $\begin{array}{c}\text { min-pid } \\
\text { (\%) }\end{array}$ & $\begin{array}{c}\text { min- } \\
\text { threshol } \\
\text { d (ratio) }\end{array}$ & $\begin{array}{c}\text { max- } \\
\text { threshol } \\
\text { d (ratio) }\end{array}$ & $\begin{array}{c}\text { min- } \\
\text { cov (\%) }\end{array}$ & $\begin{array}{c}\text { overlap } \\
\text { (nt) }\end{array}$ \\
\hline \multicolumn{1}{l}{ Autonomous TEs } & & & & & & \\
\hline LTR & 650 & $\infty$ & 95 & 0.9 & 1.1 & 95 & 5 \\
LINE & 1500 & $\infty$ & 95 & 0.9 & 1.1 & 95 & 5 \\
TIR & 700 & $\infty$ & 95 & 0.9 & 1.1 & 95 & 5 \\
Helitron & 2000 & $\infty$ & 95 & 0.9 & 1.1 & 95 & 5 \\
\hline All TEs & & & & & & & \\
\hline LTR & 650 & $\infty$ & 80 & 0.5 & 1.5 & 50 & 5 \\
LINE & 1500 & $\infty$ & 80 & 0.5 & 1.5 & 50 & 5 \\
SINE & 150 & 800 & 80 & 0.8 & 1.2 & 80 & 5 \\
TRIM & 600 & $\infty$ & 90 & 0.9 & 1.1 & 90 & 5 \\
LARD & 4000 & $\infty$ & 80 & 0.5 & 1.5 & 50 & 5 \\
TIR & 700 & $\infty$ & 80 & 0.5 & 1.5 & 50 & 5 \\
MITE & 50 & 800 & 80 & 0.85 & 1.15 & 90 & 5 \\
Helitron & 2000 & $\infty$ & 80 & 0.5 & 1.5 & 50 & 5 \\
\hline
\end{tabular}


900 Table 2: Quantity and diversity of all identified TEs. Number of TE families, number of TE copies and TEs genome coverage (\%) of all types of TEs.
Class
Order/Family
TEs identified

\section{TEs copies}

2,541

5,736

LTR/Gypsy

LTR/Unclassified

Total LTRs

LINE

SINE

TRIM

LARD

\section{Total Class I}

II

II

II

II

II

II

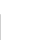

II

\section{II}

Total Class II

Total TEs

902

903

904

905

906

\section{Figure legends} file.

Figure 1. Overview of the TE Discovery pipeline. 1_Input_data: last genome assembly, available TE sequences̄ from Repbase Giri and sRNA-seq Illumina data are used as input data. 2_TEs family detection: the detection of putative TEs is performed by using four tools combining two detection approaches. The resulted sequences are merged into multi-fasta files for each type of TEs. 3_TEs clustering: Each TE type sequences are clustered with Vsearch to reduce redundancy. 4_TEs copy detection: blastn is performed to the clustered sequences with specific parameters according to each type of TE to detect copies across the genome. 5_TEs copy filter. the detected copies are subjected to filtering steps to detect "potential autonomous TEs" and/or "All TEs" including non- autonomous TEs. 6_TEs annotation: TEs annotation gff3 files are generated for each type of TEs with detailed descriptions and merged into one single

Figure 2. Comprehensive circos ideograms of TEs of the potato genome. Left panel: Retrotransposon TEs (Class I). Right panel: DNA TEs (Class II). Each concentric circle represents a different type of TE with their own color pallet and range of coverage so the distribution across the chromosomes can be appreciated. Each line within the circles 
924 represents coverage percentage per Mb. (1) Gene distribution. (a) LTR distribution. (b)

925 SINE distribution. (c) LINE distribution. (d) TRIM distribution. (e) TIR distribution. (f)

926 MITE distribution. (g) Helitron distribution.

Figure 3. Frequency histograms of TE distance to the nearest gene. Left panel:

929 Retrotransposon TEs (Class I). Right panel: DNA TEs (Class II). Bars on the left side of

930 the red line represent the TE distance within the first $5 \mathrm{~kb}$ to the nearest gene.

Figure 4. Chromosomal ideograms of LTRs age per family. Upper panel: chromosomal distribution of insertion age of all superfamilies of full-length LTR retrotransposons.

934 Lower panel: chromosomal distribution of insertion age of Gypsy, Copia and 935 Unclassified LTR retrotransposons separately.

Figure 5. Number of LTR family by insertion age according to genome chromatin state.

938 Upper panel: frequency histograms of Gypsy, Copia and Unclassified LTR families by 939 their insertion age (in millions of years) separated by heterochromatin regions (blue) and 940 euchromatic regions (red). Lower panel: Scatter plots of ten random independent 941 families from Gypsy, Copia and Unclassified LTRs assessing the age distribution of 942 individual elements by their heterochromatin (blue) and euchromatin (red) state. * shows 943 significant differences between families ( $t$-test $p<0.05$ ). 


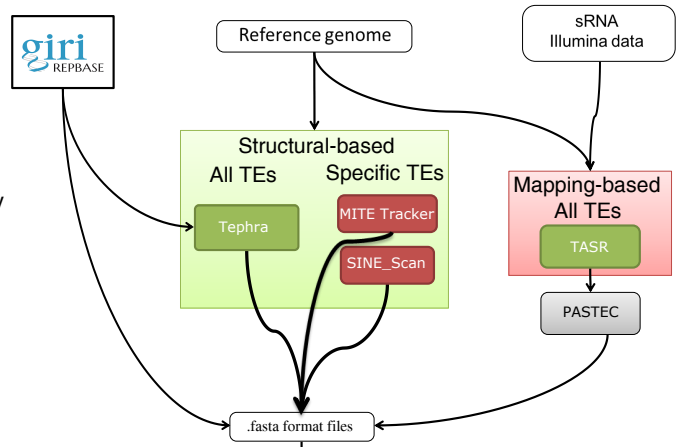

2:TEs family detection

3:TEs

clustering

4:TEs copies detection

5:TEs copies filter

6:TEs

annotation

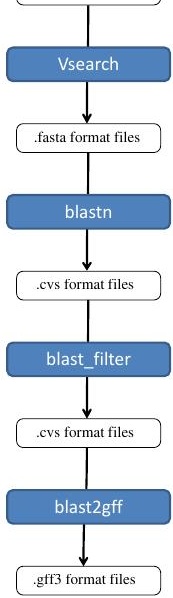




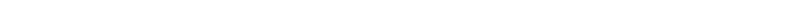




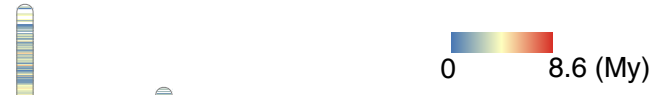

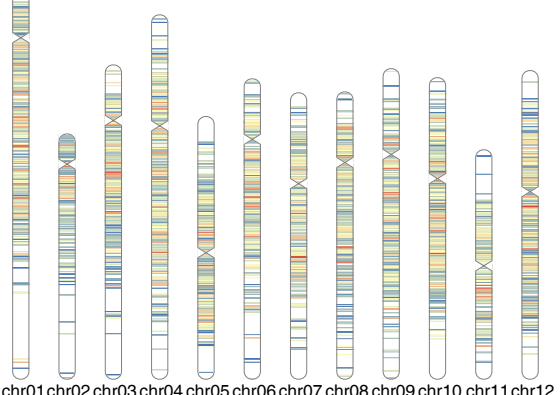

LTR Gipsy (RLG)

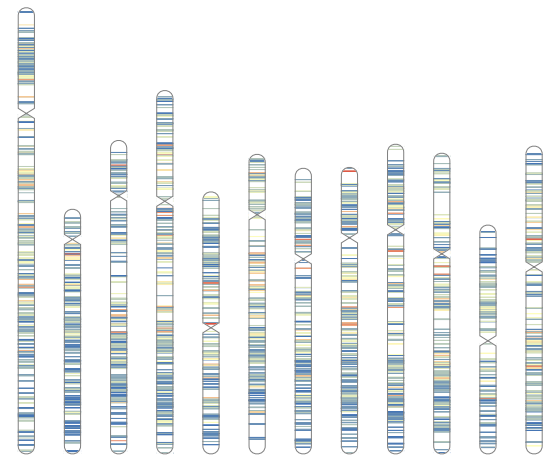

chr01 chr02 chr03 chr04 chr05 chr06 chr07 chr08 chr09 chr10 chr11 chr12

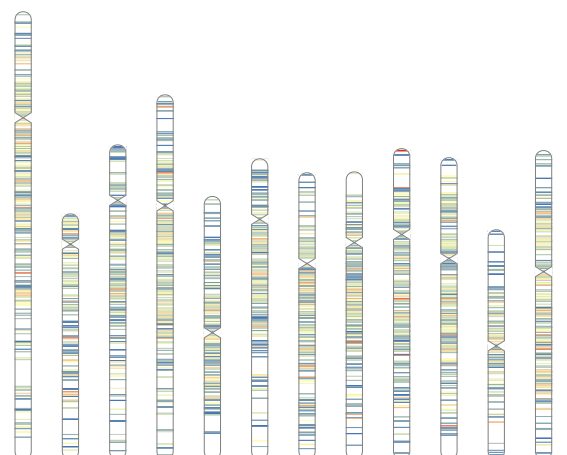

chr01 chr02 chr03 chr04 chr05 chr06 chr07 chr08 chr09 chr10 chr11 chr12 LTR Unclassified (RLX) 
